\title{
Capecitabine in the management of colorectal cancer
}

\author{
This article was published in the following Dove Press journal: \\ Cancer Management and Research \\ 23 March 20II \\ Number of times this article has been viewed
}

\section{Bradford R Hirsch \\ $S$ Yousuf Zafar}

Division of Medical Oncology, Duke University Medical Center, Durham, NC, USA
Correspondence: S Yousuf Zafar Division of Medical Oncology, Duke University Medical Center

Durham, NC 27705, USA

$\mathrm{Tel}+19196840138$

$\mathrm{Fax}+19196135228$

Email yousuf.zafar@duke.edu
Abstract: 5-Fluorouracil has been a mainstay in the treatment of colorectal cancer for nearly five decades; however, the use of oral formulations of the medication has been gaining increasing traction since capecitabine was approved for use in adjuvant settings by the US Food and Drug Administration in 2005. The use of capecitabine has since spread to a number of off-label indications, including the treatment of advanced or metastatic colorectal cancer and the neoadjuvant treatment of rectal cancer. In light of increasing utilization, it is critical that clinicians have a firm understanding of the literature supporting capecitabine across various settings as well as the attributes of the drug, such as its dosing recommendations, side-effect profile, and use in the elderly. The purpose of this review is to synthesize the literature in a fashion that can be used to help guide decisions. In a setting of increasing focus on cost, the pharmacoeconomic literature is also briefly reviewed.

Keywords: colon cancer, colorectal cancer, rectal cancer, capecitabine, Xeloda

\section{Introduction}

5-Fluorouracil (5-FU) was initially introduced over 40 years ago and has remained a mainstay in treatment regimens for colorectal cancer (CRC) since that time, both alone and in combination with other agents. Its impact on cancer care has been substantial as CRC is the third most commonly diagnosed cancer in the United States with 142,570 new cases in 2009, and it is the third leading cause of cancer death in both men and women with a combined 51,370 fatalities in the same year. ${ }^{1}$ Despite the importance of 5-FU to cancer care, its short half-life, requirement for a central line, and the need for continuous infusions led researchers to design an oral formulation of the drug. In June 2005, capecitabine (Xeloda ${ }^{\circledR}$; Hoffman-LaRoche, Nutley, NJ) was approved by the Food and Drug Administration (FDA) as an oral prodrug of 5-FU for use as monotherapy in the adjuvant setting when treating Dukes'stage C CRC.

Capecitabine has a number of advantages over traditional 5-FU. After absorption across the digestive tract, it is converted to 5-FU through three sequential enzymatic reactions. The final enzyme in the pathway, thymidine phosphorylase (TP), is believed to be present at disproportionately high levels in tumor tissue, which is said to increase both the efficacy and tolerability of the agent through targeted delivery. ${ }^{2}$ Its oral administration simplifies care, frequently precluding the need for central venous access or infusion pumps. As a result, capecitabine is increasingly used for off-label indications in $\mathrm{CRC}$, including monotherapy in the advanced or metastatic setting, combination therapy in conjunction with oxaliplatin in the advanced or metastatic setting, and with concurrent radiation for the neoadjuvant treatment of rectal cancer. ${ }^{3,4}$ As off-label use 
of capecitabine increases, it becomes even more important to understand the efficacy and tolerability across settings, which support its utilization in order to ensure the appropriate treatment of patients.

The purpose of this review is, therefore, to provide an overview of capecitabine's mechanism of action and rate of adverse events as well as an analysis of the evidence supporting its use in the settings outlined above. In addition, this article will highlight the regional differences in tolerance that affect dosing decisions and the evidence behind its use in the elderly, which remains an area of controversy. Finally, the economic literature will be discussed. The decision to prescribe capecitabine is a complex one; however, increasing evidence is emerging to guide clinicians.

\section{Methods}

For this review, English-language literature was identified through a search of PubMed (from 1966 to October 2010) and a search of The Proceedings of the American Society of Clinical Oncology (ASCO) (from January 1995 to July 2010). Search terms included capecitabine, Xeloda, colorectal cancer, colon cancer, and rectal cancer. The references of identified articles were reviewed for additional articles of interest. Studies identified as important to the field were included in the review.

\section{Mode of action and pharmacokinetics}

5-FU is an antimetabolite which disrupts DNA and RNA synthesis and repair, leading to cell death. Using folate as a cofactor, the drug is converted to active nucleotides, such as fluorodeoxyuridine monophosphate, which inhibit the enzyme thymidylate synthase. ${ }^{2,5}$ This in turn results in the formation of unbalanced pools of deoxynucleotide triphosphates that are used in DNA synthesis, leading to DNA strand breaks. Another metabolite of the drug, fluorouridine triphosphate, acts as a false nucleotide in RNA formation, inhibiting protein synthesis. ${ }^{6}$ With time, these complications result in cell death intended to arrest the progression of disease.

5-FU cannot be given orally due to significant variation in its bioavailability, leading to the design of a prodrug to overcome this drawback. The result is an oral fluoropyrimidine carbamate that mimics the serum concentrations of a continuous 5-FU infusion. Capecitabine ( $N$-(1-(5-deoxy- $\beta$ D-ribofuranosyl)-5-fluoro-1,2-dihydro-2-oxo-4-pyridinyl)- $n$ pentyl carbamate) is a crystalline substance, absorbed via the gastrointestinal tract and converted to 5-FU in three sequential enzymatic reactions. Although the first two steps occur in the liver, the final conversion from $5^{\prime}$-deoxy-5-fluorouridine to 5 -FU is believed to preferentially take place in tumor tissue because TP is expressed in higher concentrations in neoplastic tissue. ${ }^{7}$ In a study to test this hypothesis, 5-FU was found to be present at three times higher concentration in tumor tissue when compared to adjacent, normal tissue and at 21 times the concentration found in the plasma. ${ }^{8}$ In addition, a number of studies have shown that TP is upregulated in tumor tissue after treatment with radiotherapy or cytotoxic agents besides 5-FU, providing a possible explanation for the synergistic effect seen with combination therapy. ${ }^{9-12}$

Multiple studies have also assessed the pharmacokinetics of capecitabine. The drug is nearly $100 \%$ bioavailable, and a linear increase is seen in both maximum plasma concentration $\left(\mathrm{C}_{\max }\right)$ and area under the curve (AUC) with dose titration. ${ }^{13}$ There is no evidence of drug accumulation across a range of doses, and pharmacokinetics were found to be similar between Caucasian and Japanese patient populations. ${ }^{14,15}$ The half-life of capecitabine is between 0.49 and 0.89 hours, while the half-life of the metabolite (5-FU) extends from 0.67 to 1.15 hours. ${ }^{13,14,16}$ Of additional interest, capecitabine is supposed to be dosed within 30 minutes of food. A study conducted prior to capecitabine's approval compared drug levels after an overnight fast to its administration within 30 minutes of food. Researchers found a $60 \%$ decrease in the $\mathrm{C}_{\max }$ and a $31 \%$ decrease in the AUC when given with food, but the change in AUC of the cytotoxic end product, 5-FU, exhibited less variation. ${ }^{17}$ The importance of taking the medication with food is, therefore, unclear, but it is still recommended to be taken within 30 minutes of a meal, as this was the setting in which it was tested.

\section{Dosing}

The ideal dosing of capecitabine is controversial as regional differences have been seen in the tolerance of oral fluoropyrimidines. In 2008, Haller et al published a retrospective analysis of patients with CRC who were treated with capecitabine at sites around the world. ${ }^{18}$ Among 1189 patients with metastatic disease, the authors found that those enrolled in trials in the United States had higher rates of grade 3 and 4 adverse events (relative risk [RR], 1.77), an increase in the frequency of dose reduction ( $R R, 1.72)$, and higher rates of treatment discontinuation ( $R R, 1.83)$. The results among 1864 patients receiving treatment in the adjuvant setting also showed increased grade 3 and 4 adverse events (RR, 1.47) and higher rates of discontinuation (RR, 2.09). On further analysis, East Asian patients fared the best overall.

This was again demonstrated in the TREE trials. ${ }^{19}$ In the first of two (TREE-1), 150 patients were treated with 
a combination of capecitabine and oxaliplatin (XELOX) at a capecitabine dose of $1000 \mathrm{mg} / \mathrm{m}^{2}$. In the subsequent trial (TREE-2), the capecitabine dose was reduced to $850 \mathrm{mg} / \mathrm{m}^{2}$ for the 223 enrolled patients. The dose reduction was made after the data monitoring committee reviewed the safety data of the initial study. In TREE-2, an increase in hypertension was seen due to the addition of bevacizumab, while the overall rate of grade 3 and 4 toxicities improved as shown in Table 1.

A number of possible explanations for the variation in side effect profiles between countries have been proposed. Folic acid supplementation is much more widespread in the United States than in Europe which might account for a portion of the differences. Pharmacogenetics may also play a role as genetic differences between Caucasian and Japanese patients have been discovered, but this is unlikely to explain the variation in events between the United States and European populations who have similar genetic profiles. ${ }^{20}$ Variability in trial reporting, psychosocial factors, and differences in body weight have also been considered as causative factors in the regional toxicity variation, but none have been substantiated.

Capecitabine was initially approved at a dose of $2500 \mathrm{mg} / \mathrm{m}^{2}$ for 14 of every 21 days; however, this dose results in increased toxicity in the United States as outlined above. The most recent National Comprehensive Cancer Network guidelines recommend starting doses of 850 or $1000 \mathrm{mg} / \mathrm{m}^{2}$ twice daily when used with oxaliplatin for advanced or metastatic colorectal cancer ( $\mathrm{mCRC}$ ), while the group recommends using $1000-1250 \mathrm{mg} / \mathrm{m}^{2}$ twice daily when it is given as monotherapy. ${ }^{3}$ With concurrent radiation for rectal cancer, the recommended dose is $850 \mathrm{mg} / \mathrm{m}^{2}$ twice daily. ${ }^{4}$

\section{Treatment strategies}

Capecitabine is used to treat CRC in three settings: for adjuvant treatment, as monotherapy or in combination with

Table I Incidence of grade 3 and 4 adverse events (\%) in mCRC treatments $^{19}$

\begin{tabular}{lll}
\hline Parameter & TREE-I $(\mathbf{n}=\mathbf{1 5 0})$ & TREE-2 $(\mathbf{n}=\mathbf{2 2 3})$ \\
\hline Nausea/vomiting & 38 & 21 \\
Diarrhea & 31 & 19 \\
Dehydration & 27 & 8 \\
Paresthesia & 21 & 11 \\
Hand-foot syndrome & 19 & 10 \\
Fatigue & 6 & 11 \\
Anemia & 6 & 0 \\
Leukopenia & 2 & 0 \\
Hypertension & 2 & 15
\end{tabular}

Adapted from Hochster et a $\left.\right|^{19}$, with permission $\odot 2008$ American Society of Clinical Oncology.

Abbreviation: $\mathrm{mCRC}$, metastatic colorectal cancer. other agents for advanced or metastatic disease, and with concurrent radiation for the neoadjuvant treatment of rectal cancer. The most robust body of literature addresses its role in metastatic disease, but the literature across all indications is increasing.

\section{Adjuvant treatment of CRC}

Capecitabine was initially approved by the FDA in 2005 for treatment of Dukes' $\mathrm{C}$ (now commonly referred to as stage III) CRC in the adjuvant setting. This approval was based largely on results from the Xeloda in Adjuvant Colon Cancer Therapy (X-ACT) trial in which 1987 patients with previously resected stage III colon cancer were randomized to either capecitabine at $1250 \mathrm{mg} / \mathrm{m}^{2}$ (1004 patients) twice daily or bolus 5-FU plus leucovorin via the Mayo regimen (983 patients). ${ }^{21}$ At a median of 3.8 years of follow-up, the authors concluded that capecitabine demonstrated noninferiority to 5-FU. Although relapse-free survival was significantly improved (hazard ratio [HR], 0.86; 95\% confidence interval [CI]: 0.74-0.99), differences in disease-free survival (DFS) (HR, 0.87; 95\% CI: 0.75-1.00) and overall survival (OS) (HR, 0.84; 95\% CI: 0.69-1.01) bordered on statistical significance. Of interest, $83 \%$ of patients receiving capecitabine completed their treatment course, but 57\% required dose modifications. Overall, the toxicity profile of capecitabine was superior with the exception of a greater frequency of hand-foot syndrome (HFS). As a result, capecitabine is generally considered to be noninferior to 5-FU in the adjuvant setting, supporting its use among those patients who chose to undergo treatment.

A number of ongoing studies are investigating capecitabine in a variety of adjuvant settings. Preliminary results from N016968 demonstrated significant increases in 3-, 4-, and 5-year DFS rates for stage III patients receiving XELOX when compared to 5-FU and leucovorin. ${ }^{22}$ A large European study of the adjuvant treatment of CRC (AVANT) is comparing XELOX plus bevacizumab to either FOLFOX alone or FOLFOX with bevacizumab. It is yet to release final results, but initial indications suggest no differences in outcomes between the different regimens. ${ }^{23}$ The European PETACC-6 study is comparing neoadjuvant capecitabine and radiation, followed by surgery and adjuvant capecitabine to the same regimen plus oxaliplatin in patients with rectal cancer. ${ }^{24} \mathrm{~A}$ phase III study enrolling in the United Kingdom is examining the benefit of adjuvant XELOX in locally advanced rectal cancer. ${ }^{25}$ These studies suggest a growing interest in capecitabine in a variety of regimens in the adjuvant setting. However, since no definitive data are presently 
available, FOLFOX remains the regimen of choice over XELOX when combination treatment is being considered in the adjuvant setting. For patients who are resistant to carrying a pump, the consideration of capecitabine or XELOX is within reason.

\section{Concurrent chemotherapy and radiation in rectal cancer}

Preoperative chemotherapy and radiation have become the standard of care with T3 and T4 rectal cancer as it results in lower rates of local recurrence when compared to adjuvant chemotherapy and radiation. The regimen used by the German Rectal Cancer Study Group, which first demonstrated the role of neoadjuvant treatment, consists of two doses of infusional 5-FU on weeks 1 and 5. ${ }^{26}$ This has been routinely used since that time; however, capecitabine has recently been gaining traction as an off-label alternative to 5-FU. Although preclinical studies showed promise that capecitabine could be more effective than 5-FU due to the upregulation of $\mathrm{TP}^{24}$ the quality of evidence supporting this practice is not robust.

A number of trials have been performed looking at monotherapy with capecitabine, and a summary of the phase II trials is shown in Table 2. A range of downstaging following treatment has been found to be between $51 \%$ and $76 \%$ in these studies, with a complete response seen on pathology between $12 \%$ and $31 \%$ of the time. ${ }^{27-30}$ The largest of these studies was a trial of 95 patients in South Korea with T3, T4, or N1 disease. They were treated with capecitabine at $825 \mathrm{mg} / \mathrm{m}^{2}$ twice daily while receiving 50 Gy of radiation followed by a total mesorectal excision. ${ }^{30}$ This trial was not randomized or blinded and patients were compared to historical controls. Out of 95 patients enrolled, $97 \%$ were able to undergo complete resection, $76 \%$ of the tumors were downstaged at the time of surgery, and $12 \%$ had a complete response on pathologic evaluation. These results were said to be consistent with historical results seen with 5-FU; however, the trial results did not include outcome data such as progression-free survival (PFS) or OS.

Another important study which has only been presented in abstract form, is a Phase III trial from Germany which randomized patients receiving 50.4 Gy of radiation to concurrent infusional $5-\mathrm{FU}$ or capecitabine at $825 \mathrm{mg} / \mathrm{m}^{2}$ twice daily. ${ }^{31}$ The initial results have been presented on 121 patients. Those treated with capecitabine had higher rates of tumor downstaging $(52 \%$ vs $39 \% ; P=0.16)$ and increased N0 status ( $71 \%$ vs $56 \% ; P=0.09)$; however, neither result was statistically significant. As expected, the patients receiving capecitabine had less leukopenia ( $25 \%$ vs $35 \%$; $P=0.04$ ) but more HFS $(31 \%$ vs $2 \% ; P<0.001)$. The toxicity profiles were otherwise similar.

A number of trials have also looked at the addition of oxaliplatin, irinotecan, or targeted agents to chemoradiation with capecitabine. The results from phase II and III trials on the subject are also outlined in Table 2. The possible utility of oxaliplatin was extrapolated from the colon cancer setting where a radiosensitizing effect and DFS benefit have been shown. Among phase II studies, downstaging ranged from $53 \%$ to $67 \%$ with complete responses seen at pathologic evaluation $14 \%$ to $20 \%$ of the time. ${ }^{32-36}$ However, a phase III study showed only a nonsignificant gain in complete response despite a significant increase in toxicity. In a trial by Chua et al, they amended the protocol to exclude patients with cardiac disease after deaths in 8 of an initial 77 patients receiving the regimen. ${ }^{33}$ This causes concern for an otherwise promising regimen.

Irinotecan has less evidence supporting its use, in part because of overlapping toxicities. Although two small phase I/II studies have been performed which show complete response rates of $15 \%$, the rate of grade 3 or 4 diarrhea was $20 \%$ in one of the studies and the rate of grade 3 or 4 leukopenia was $25 \%$ in the other. ${ }^{37,38}$ As such, this cannot be considered a viable alternative at present. Although studies have also looked at targeted therapies, including cetuximab and bevacizumab, the results are not yet robust enough to make clinical decisions. Trials including phase II arms are again outlined in Table 2. ${ }^{39,40}$

Trials assessing the treatment of rectal cancer with concurrent capecitabine and radiation are promising, but the comparability between 5-FU and capecitabine in this setting has not been definitively proven. An ongoing trial, NSABP R-04, will shed more light on the topic as patients are enrolled in a two-by-two factorial design in order to compare capecitabine to infusional 5-FU, with or without oxaliplatin. The final publication of this study as well as the German trial mentioned above should help shed light on the topic. Based on the currently available evidence, capecitabine can be reasonably considered in patients who are reluctant to receive continuous infusion therapy during the course of radiation.

\section{Advanced and $\mathrm{mCRC}$}

The use of capecitabine in the advanced or metastatic settings is of interest, as quality of life is particularly important in patients who are often unlikely to be cured of their disease. In a recent analysis of patient preference, it was found that $95 \%$ preferred oral palliative chemotherapy prior to starting 


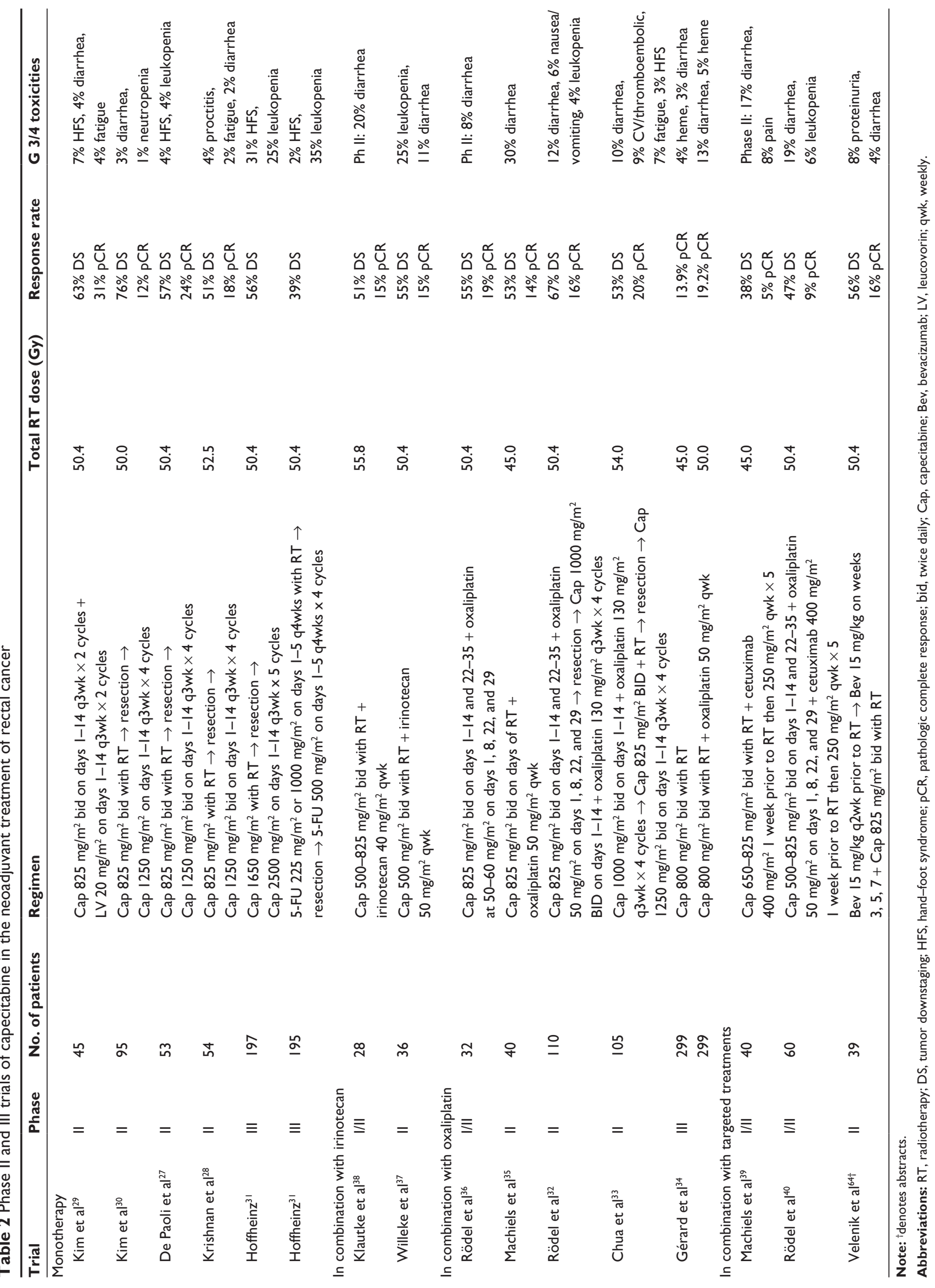


treatment, and $64 \%$ retained this preference after treatment. ${ }^{41}$ This is consistent with prior research on the matter; however, $70 \%$ of patients have stated that they are not willing to do so if it meant a lower response rate. ${ }^{42} \mathrm{~A}$ meta-analysis published in October 2010 found 22 trials comparing capecitabine-based regimens to treatment with 5 -FU in the metastatic setting. ${ }^{43}$ A total of 12 studies was excluded from the analysis, most often due to a lack of randomization, and the results included both monotherapy and combination regimens. Although the analysis found a significant improvement in PFS favoring capecitabine, the gain in OS was not significant (RR, 0.89; 95\% CI: 0.73-1.09).

It is important to look at the specific combinations to help inform decisions. To evaluate capecitabine as monotherapy in advanced disease, two phase III trials were conducted at a dose of $1250 \mathrm{mg} / \mathrm{m}^{2}$ twice daily for 2 of every 3 weeks. In both trials, capecitabine was compared to the Mayo Clinic regimen of bolus 5-FU and leucovorin, which differs from the continuous infusion protocol used in most modern regimens. ${ }^{44,45}$ The studies showed improved response rates favoring capecitabine ( $18.9 \%$ vs $15.0 \%$ and $24.8 \%$ vs $15.5 \%$ ); however, these results were not statistically significant, and both time to progression and OS were not significantly different. Patients treated with capecitabine exhibited lower rates of neutropenia, stomatitis, and alopecia but had higher rates of cutaneous HFS and uncomplicated hyperbilirubinemia.

These studies have been supplemented with numerous others looking at the role of capecitabine as a replacement for 5-FU and leucovorin in either the FOLFOX (5-FU, leucovorin, and oxaliplatin) or FOLFIRI (5-FU, leucovorin, and irinotecan) regimens. Of note, after substituting capecitabine in place of 5-FU, the combinations are instead referred to as XELOX and CAPOX or XELIRI and CapeIRI. The dosing of the different agents in the regimens is outlined in the footnote to Table 3.

Numerous phase III trials have looked at the differences between FOLFOX and XELOX in first- and second-line settings, some of which have been published only in abstract form. ${ }^{46-50}$ These trials are again outlined in Table 3. All of the trials demonstrated XELOX to have similar outcomes to FOLFOX, including rates of response, PFS, and OS. None found XELOX to be superior, and most have actually shown that the survival results for XELOX lag those of FOLFOX without being statistically significant.

Trial NO16966 was the most robust assessment of the topic. ${ }^{46}$ It was Roche-sponsored and compared XELOX to FOLFOX4 for first-line therapy in the advanced or metastatic setting. It initially included 634 patients; however, an additional 1400 patients were added after the protocol was altered to allow two-by-two randomization with the addition of bevacizumab. Among the 2034 patients enrolled, there was no significant difference in primary outcomes seen between the XELOX and FOLFOX4 arms, as PFS was found to be 8.0 and 8.5 months (HR, 1.04; 95\% CI: 0.93-1.16), respectively, while OS were 19.8 and 19.6 months (HR, 0.99; 95\% CI: 0.88-1.12). The authors concluded that XELOX is noninferior for the up-front treatment of metastatic disease.

Multiple studies have also reported on the combination of capecitabine and irinotecan, as outlined in Table 3. The CAIRO study investigated the benefit of sequential versus combination therapy using capecitabine, oxaliplatin, and irinotecan. ${ }^{51}$ Patients receiving CapeIRI in this trial reportedly experienced gastrointestinal toxicity within an acceptable range. However, two subsequent studies reported contrasting findings. The phase III BICC-C trial compared FOLFIRI, CapeIRI, and irinotecan with bolus 5-FU (mIFL). ${ }^{52}$ Capecitabine was dosed at $1000 \mathrm{mg} / \mathrm{m}^{2}$ twice daily. In this study, FOLFIRI was found to be superior to either alternative with a median PFS of 7.6 months compared to 5.9 months for mIFL $(P=0.004)$ and 5.8 months for CapeIRI $(P=0.015)$. OS results trended toward superiority at 23.1 months for FOLFIRI, 17.6 months for mIFL $(P=0.09)$, and 18.9 months for CapeIRI ( $P=0.27)$; however, none of the results achieved significance. CapeIRI also had higher rates of grade 3 and 4 diarrhea (48\%), dehydration (19\%), nausea (18\%), and vomiting (16\%). The authors considered the possibility that worse PFS with CapeIRI was attributable to treatment discontinuation from toxicity. The data were reanalyzed after excluding those patients who discontinued treatment within 30 days due to toxicity, and PFS for CapeIRI was still inferior to that of FOLFIRI.

A third trial was designed to show noninferiority of CapeIRI to FOLFIRI in the first-line metastatic setting (EORTC 40015). ${ }^{53}$ In this two-by-two study, patients were randomized to receive celecoxib or placebo in addition to chemotherapy. Of note, this trial was closed after enrollment of only 85 of a planned 692 patients due to seven deaths not related to disease progression (five in the CapeIRI arm and two in the FOLFIRI arm). Analysis of this markedly limited data demonstrated worse outcomes with CapeIRI than FOLFIRI and worse outcomes with celecoxib than placebo. The results of these trials, and considerable overlap noted between the toxicity profiles of capecitabine and irinotecan, have led to concern about the use of these two drugs in combination.

Increasing evidence suggests that capecitabine is noninferior as monotherapy and in combination with oxaliplatin when compared to 5-FU in the advanced and metastatic 
Table 3 Published phase III trials involving capecitabine in $\mathrm{mCRC}$

\begin{tabular}{|c|c|c|c|c|c|c|c|}
\hline Author & Regimen & $\begin{array}{l}\text { Line of } \\
\text { treatment }\end{array}$ & $\begin{array}{l}\text { No. of } \\
\text { patients }\end{array}$ & ORR (\%) & $\begin{array}{l}\text { Median } \\
\text { PFS (m) }\end{array}$ & $\begin{array}{l}\text { Median } \\
\text { OS (m) }\end{array}$ & G $3 / 4$ toxicities \\
\hline \multicolumn{8}{|c|}{ Comparisons of single agent capecitabine to 5-FU/LV (Mayo) } \\
\hline \multirow[t]{2}{*}{ Hoff et al ${ }^{44}$} & Capecitabine & I & 302 & $24.8^{*}$ & 4.3 & 12.5 & $\begin{array}{l}15.4 \% \text { diarrhea, } \\
18 \% \text { HFS, } 3 \% \text { S }\end{array}$ \\
\hline & $5-\mathrm{FU}$ & & 303 & 15.5 & 4.7 & 13.3 & $\begin{array}{l}\text { 13.9\% diarrhea, } \\
1 \% \mathrm{HFS}, 16 \% \mathrm{~S}\end{array}$ \\
\hline \multirow[t]{2}{*}{ Van Cutsem et al ${ }^{45}$} & Capecitabine & I & 301 & 18.9 & 5.2 & 13.2 & $\begin{array}{l}10.7 \% \text { diarrhea, } \\
16 \% \text { HFS, } 1 \% \text { S }\end{array}$ \\
\hline & $5-\mathrm{FU}$ & & 301 & 15 & 4.7 & 12.1 & $\begin{array}{l}10.4 \% \text { diarrhea, } \\
<1 \% \mathrm{HFS}, 13 \% \mathrm{~S}\end{array}$ \\
\hline \multicolumn{8}{|c|}{ Comparisons of oxaliplatin containing regimens } \\
\hline \multirow[t]{2}{*}{ Díaz-Rubio et a ${ }^{48}$} & XELOX & I & 174 & 37 & 8.9 & 18.1 & $\begin{array}{l}14 \% \text { diarrhea, } \\
2 \% \text { HFS, } 2 \% \text { S }\end{array}$ \\
\hline & FUOX & & 174 & 46 & 9.5 & 20.8 & $\begin{array}{l}24 \% \text { diarrhea, } \\
1 \% \mathrm{HFS}, 4 \% \mathrm{~S}\end{array}$ \\
\hline \multirow[t]{2}{*}{ Porschen et $\mathrm{al}^{47}$} & CAPOX & 1 & 242 & 48 & 7.1 & 16.8 & $10 \% \mathrm{HFS}$ \\
\hline & FUFOX & & 234 & 54 & 8.0 & 18.8 & $4 \% \mathrm{HFS}$ \\
\hline \multirow[t]{2}{*}{$\begin{array}{l}\text { Cassidy et al }{ }^{46} \\
(\mathrm{NO} 16966)\end{array}$} & XELOX & 1 & 1017 & 37 & 8.0 & 19.8 & $\begin{array}{l}11 \% \text { diarrhea, } \\
6 \% \mathrm{HFS}, 1 \% \mathrm{~S}\end{array}$ \\
\hline & FOLFOX4 & & 1017 & 37 & 8.5 & 19.6 & $\begin{array}{l}20 \% \text { diarrhea, } \\
1 \% \text { HFS, } 2 \% \text { S }\end{array}$ \\
\hline \multirow{3}{*}{$\begin{array}{l}\text { Hochster et al }{ }^{19} \\
\text { (TREE-I) }\end{array}$} & CapeOX & 1 & 50 & 27 & 5.9 & 17.2 & Refer to Table I \\
\hline & mFOLFOX6 & & 50 & 41 & 8.7 & 19.2 & \\
\hline & bFOL & & 50 & 20 & 6.9 & 17.9 & \\
\hline \multirow[t]{2}{*}{ Rothenberg et $\mathrm{al}^{50}$} & XELOX & II & 313 & 20 & 4.7 & 11.9 & $\begin{array}{l}5 \% \text { diarrhea, } \\
\text { HFS } 4 \%, S<1 \%\end{array}$ \\
\hline & FOLFOX4 & & 314 & 18 & 4.8 & 12.5 & $\begin{array}{l}19 \% \text { diarrhea, } \\
\text { HFS }<1 \%, \mathrm{~S}<1 \%\end{array}$ \\
\hline \multicolumn{8}{|c|}{ Comparison of irinotecan containing regimens } \\
\hline \multirow[t]{3}{*}{$\begin{array}{l}\text { Fuchs et al }{ }^{52} \\
\text { (BICC-C) }\end{array}$} & CapelRI & I & 145 & 39 & 5.8 & 18.9 & $\begin{array}{l}48 \% \text { diarrhea, } \\
32 \% \text { neutropenia }\end{array}$ \\
\hline & FOLFIRI & & 144 & 47 & $7.6^{*}$ & 23.1 & $\begin{array}{l}14 \% \text { diarrhea, } \\
43 \% \text { neutropenia }\end{array}$ \\
\hline & $\mathrm{mlFL}$ & & 141 & 43 & 5.9 & 17.6 & $\begin{array}{l}19 \% \text { diarrhea, } \\
41 \% \text { neutropenia }\end{array}$ \\
\hline \multirow[t]{2}{*}{$\begin{array}{l}\text { Koopman et al }{ }^{51} \\
\text { (CAIRO) }\end{array}$} & Cap/IRI/CAPOX & I-III & 410 & 20 & 5.8 & 16.3 & $\begin{array}{l}\text { 23\% diarrhea, } \\
13 \% \text { HFS 3\% S }\end{array}$ \\
\hline & CAPIRI/CAPOX & & 410 & $41^{*}$ & $7.8^{*}$ & 17.4 & $\begin{array}{l}27 \% \text { diarrhea, } \\
7 \% \text { HFS, } 2 \% \text { S }\end{array}$ \\
\hline \multirow[t]{2}{*}{$\begin{array}{l}\text { Köhne et al }{ }^{53} \\
\text { (EORTC 400I5) }\end{array}$} & CapelRI & I & 44 & 34 & 5.9 & 14.8 & $\begin{array}{l}37 \% \text { diarrhea, } 14 \% \\
\text { neutropenia, } 9 \% \mathrm{CV}\end{array}$ \\
\hline & FOLFIRI & & 41 & 39 & $9.6^{*}$ & $19.9 *$ & $\begin{array}{l}13 \% \text { diarrhea, } \\
15 \% \text { neutropenia }\end{array}$ \\
\hline
\end{tabular}

Notes: *Achieved statistical significance. Capecitabine combinations: CapelRI: capecitabine $1000 \mathrm{mg} / \mathrm{m}^{2}$ twice daily (bid) on days $\mathrm{I}-\mathrm{I} 4+\mathrm{irinotecan} 250 \mathrm{mg} / \mathrm{m}^{2} \mathrm{q} 3 \mathrm{wk}$; CapeOX: capecitabine $1000 \mathrm{mg} / \mathrm{m}^{2}$ bid on days $\mathrm{I}-15$ and oxaliplatin $130 \mathrm{mg} / \mathrm{m}^{2}$ on DI q3wk; XELOX: capecitabine $1000 \mathrm{mg} / \mathrm{m}^{2}$ bid on days $1-14$ and oxaliplatin I $30 \mathrm{mg} / \mathrm{m}^{2}$ on DI q3wk. 5-FU combinations: bFOL: oxaliplatin $85 \mathrm{mg} / \mathrm{m}^{2}$ on DI and DI5 + 5-FU $500 \mathrm{mg} / \mathrm{m}^{2}+\mathrm{LV} 20 \mathrm{mg} / \mathrm{m}^{2}$ on DI, D8, DI5 q4wk; FOLFIRI: irinotecan I80 mg/m², LV $400 \mathrm{mg} / \mathrm{m}^{2}, 5$-FU $400 \mathrm{mg} / \mathrm{m}^{2}$ bolus then 5 -FU $2400 \mathrm{mg} / \mathrm{m}^{2}$ over $46 \mathrm{~h}$ q2wk; FUFOX: oxaliplatin $50 \mathrm{mg} / \mathrm{m}^{2}$, LV $500 \mathrm{mg} / \mathrm{m}^{2}$, and 5-FU $2000 \mathrm{mg} / \mathrm{m}^{2}$ over $22 \mathrm{~h}$ on DI, D8, DI5, D22 q36 days; FUOX: continuous infusion 5-FU $2250 \mathrm{mg} / \mathrm{m}^{2}$ over $48 \mathrm{~h}$ on DI, D8, DI5, D22, D29, D36 + oxaliplatin $85 \mathrm{mg} / \mathrm{m}^{2}$ on DI, DI5, D29; FOLFOX4: oxaliplatin $85 \mathrm{mg} / \mathrm{m}^{2}$, LV $200 \mathrm{mg} / \mathrm{m}^{2}, 5-\mathrm{FU} 400 \mathrm{mg} / \mathrm{m}^{2}$ bolus then $5-\mathrm{FU} 600 \mathrm{mg} / \mathrm{m}^{2}$ over $22 \mathrm{~h}$ on DI, D2 q2wk; mFOLFOX6: oxaliplatin $85 \mathrm{mg} / \mathrm{m}^{2}+\mathrm{LV} 350 \mathrm{mg} / \mathrm{m}^{2}, 5-\mathrm{FU} 400 \mathrm{mg} / \mathrm{m}^{2}$ bolus then 5-FU $2400 \mathrm{mg} / \mathrm{m}^{2}$ 46-h infusion q2wk.

Abbreviations: HFS, hand-foot syndrome; S, stomatitis; MCRC, metastatic colorectal cancer; ORR, overall response rate; OS, overall survival; PFS, progression-free survival; LV, leucovorin.

settings. The same cannot be said definitively for its use with irinotecan due to worse outcomes and toxicity. The combination of capecitabine and irinotecan should be used with caution, and dose reductions should be considered with early signs of toxicity.

\section{Adverse events, elderly patients, and other considerations}

The side effect profile of capecitabine varies from that of 5-FU. In a review of 750 patients treated for colorectal or breast cancer, $>25 \%$ of patients experienced anemia, 
diarrhea, HFS, nausea, hyperbilirubinemia, fatigue/weakness, abdominal pain, vomiting, and dermatitis. ${ }^{54}$ Among the 596 CRC patients in the study, the most common grade 3 toxicities included hyperbilirubinemia (18\%), HFS (17\%), diarrhea (13\%), abdominal pain (9\%), nausea (4\%), vomiting (4\%), ileus (4\%), and fatigue/weakness (4\%). Grade 4 toxicities occurring in more than $1 \%$ of patients included hyperbilirubinemia (5\%), neutropenia (2\%), and diarrhea (2\%). As discussed earlier, dosing schedules can play a significant role in the rate of complications.

In CRC patients, it is particularly important to compare the safety profile of capecitabine with 5-FU. In an analysis of phase III trials by Walko and Lindley in 2005, the most common toxicities for both capecitabine and 5-FU were fatigue (21.1\% and $25 \%$ ) and vomiting (23.3\% and 27\%). ${ }^{54}$ Capecitabine resulted in a higher rate of HFS (53.3\% vs 6.2\%), while 5 -FU increased the rate of stomatitis $(24.3 \%$ vs $61.6 \%)$, alopecia $(6.0 \%$ vs $20.6 \%)$, neutropenia ( $1.2 \%$ vs $10.3 \%)$, diarrhea (47.7\% vs 58.2\%), and nausea (37.9\% vs 47.6\%). In a recent meta-analysis of mCRC trials by Ling et al, similar findings were seen when comparing the toxicity profiles of capecitabine and 5-FU as outlined in Table $4 .{ }^{43}$ It is, therefore, important to discuss the different side effects with patients, explaining that the overall profiles appear to favor capecitabine.

Treatment of elderly patients with capecitabine has been a particular focus of recent research due to concerns about their ability to tolerate treatment. An abstract presented at ASCO in 2004 compared the rate of adverse events among patients who were aged between 41 and 60 years with patients aged above 70 years. ${ }^{55}$ The analysis found that the overall safety profiles favored capecitabine over 5-FU, with similar toxicities across age groups taking capecitabine. A trial of 51 patients aged above 70 years found similar results with $67 \%$ achieving disease control and only $12 \%$ of patients exhibiting grade 3 or 4 toxicity on capecitabine. ${ }^{56}$

The FOCUS2 trial results were published in April 2010, specifically looking at the treatment of elderly patients

Table 4 Comparison of grade 3 and 4 events in patients treated with capecitabine in trials as opposed 5-FU per a recent meta-analysis of $\mathrm{mCRC}$ trials $^{43}$

\begin{tabular}{lllll}
\hline G 3/4 AE & No. of trials & No. of patients & OR & $\mathbf{9 5 \%} \mathbf{C l}$ \\
\hline Thrombocytopenia & 6 & 2612 & 1.45 & $0.82-2.55$ \\
Diarrhea* & 10 & 4720 & 1.35 & $1.16-1.57$ \\
Nausea/vomiting & 8 & 4668 & 1.06 & $0.84-1.33$ \\
Neuropathy & 7 & 4525 & 1.04 & $0.82-1.32$ \\
Neutropenia* & 9 & 4786 & 0.15 & $0.12-0.18$ \\
\hline
\end{tabular}

Note: *Statistically significant.

Abbreviations: 5-FU, 5-fluorouracil; $m C R C$, metastatic colorectal cancer; $A E$, adverse events; $\mathrm{OR}$, odds ratio; $\mathrm{Cl}$, confidence interval. with capecitabine and bevacizumab as a first-line regimen for mCRC. ${ }^{57}$ A total of 59 European patients aged above 70 years were enrolled. Results showed a 34\% response rate with $71 \%$ of patients achieving disease control at a dose of $1250 \mathrm{mg} / \mathrm{m}^{2}$ twice daily. A total of $54 \%$ of patients had grade 3 or 4 toxicities and four patients died due to treatment-related complications. A higher rate of grade 3 and 4 toxicities was seen in patients with decreased renal clearance. A trial in Italy compared CAPOX with CAPIRI among 94 patients aged above 70 years. ${ }^{58}$ Similar outcomes were seen in both groups; however, CAPOX was better tolerated with less grade 3 or 4 diarrhea ( $32 \%$ vs $15 \%$ ), less neutropenia ( $23 \%$ vs $6 \%$ ), and an improved global health status (45\% vs $21 \%$ ).

These trials indicate that capecitabine can be administered to patients aged above 70 as long as they desire aggressive care and are comfortable with the rate of adverse reactions outlined. Capecitabine should be avoided in elderly patients with renal dysfunction or overlapping toxicities.

Another concern has been the increased toxicity seen in patients treated with capecitabine after prior exposure to 5-FU. ${ }^{59} \mathrm{~A}$ trial randomizing patients to sequential treatment with weekly bolus 5-FU followed by capecitabine or capecitabine followed by 5-FU was closed after accruing 40 of the planned 74 patients because of excessive sequence-specific toxicity. The mechanism behind this finding is unclear, and it is not clear if this would still be seen with a longer delay between exposures to these agents. Of note, this data is very limited and does not necessarily apply to modern infusional 5-FU regimens.

\section{Pharmacoeconomics}

Reimbursement policies vary internationally and even regionally within the United States. Multiple analyses have shown cost savings when capecitabine is compared to 5-FU, but variability in insurance coverage for oral cytotoxics can lead to significantly higher out-of-pocket expenses for patients, especially since 5-FU is usually fully covered by insurance plans.

In a 2009 article by $\mathrm{Chu}$ et al, patients treated with capecitabine for $\mathrm{CRC}$ were assessed for the frequency and expense of a range of common complications. ${ }^{60}$ Among 4973 patients, the mean predicted monthly complication cost was $136 \%$ greater with 5-FU monotherapy when compared to capecitabine. This equated to an additional US\$601/month (95\% CI: \$469-\$737) spent treating complications associated with 5-FU. When each agent was given in combination with oxaliplatin, this value increased to US\$1165/month (95\% CI: \$892-\$1595). The costs of administering the drugs were not included in the calculation as both values were similar. 
An analysis of the X-ACT trial (which evaluated capecitabine versus 5 -FU in the adjuvant treatment of CRC) showed the cost of treating patients with capecitabine in the United Kingdom was $57 \%$ lower than that for 5-FU. ${ }^{61}$ Capecitabine use led to decreased hospitalization rates and cost savings of $£ 3653$. Societal costs for such things as patient travel and time off work were also lowered by $£ 1318$, again reinforcing the potential cost benefit of capecitabine.

An analysis comparing costs between XELOX and FOLFOX4 as a part of trials NO16966 and NO16967 was also reported in 2009. ${ }^{62}$ In the analysis, the authors found the incremental improvement in quality-adjusted progression free survival days (QAPFSD) favored XELOX over FOLFOX4 in both first- and second-line settings. Specifically, patients gained 10.5 QAPFSD from first-line and 11.3 QAPFSD from secondline treatment. Cost calculations found savings for the National Health Service of $£ 7600$ and $£ 3900$ for patients treated with XELOX in first- and second-line settings, respectively.

Finally, a study published in the United States demonstrated a lower cost with capecitabine monotherapy when compared to 5-FU and leucovorin of US\$6683 versus US\$9304. ${ }^{63}$ It showed a higher acquisition cost for capecitabine but lower administration and complication costs. This held true when oxaliplatin was added to the regimens with costs of US $\$ 11,463$ and US $\$ 14,320$. Overall, these studies demonstrate a measurable cost saving when using capecitabine in place of 5-FU. However, most of these studies are limited in that the overall costs to the patient, provider, and payer are not considered in the calculation.

\section{Conclusion}

In the past decade, capecitabine has been heavily investigated in all CRC treatment settings. Although it was initially approved for use by the FDA in the adjuvant setting for stage III disease, the most robust data exist in the metastatic setting. Its off-label use is quickly growing and will continue to do so, pending the publication of a number of ongoing clinical trials, especially in the adjuvant and neoadjuvant settings. For metastatic disease, the evidence is fairly robust, showing capecitabine to be noninferior to 5-FU as monotherapy or as a part of a combined regimen with oxaliplatin. The same cannot be said for combinations with irinotecan as overlapping toxicity profiles lead to poor tolerability, and studies have had trouble demonstrating equivalent outcomes. In the adjuvant setting, the X-ACT trial established borderline superiority to treatment with capecitabine, while a Japanese meta-analysis showed noninferiority when compared to observation alone. This is encouraging for capecitabine use. Studies with capecitabine in the neoadjuvant setting have at least demonstrated efficacy and tolerability, but two major ongoing studies will hopefully shed more light on capecitabine in direct comparison to 5-FU with radiation in the neoadjuvant treatment of rectal cancer.

In addition to disease outcomes such as PFS and OS, patients should be informed about differences in the side effect profiles between capecitabine and 5-FU and out-of-pocket costs. Depending on insurance coverage, capecitabine use in place of 5-FU may be less expensive for the system as a whole but may result in significantly higher out-of-pocket costs for a particular individual. Extra care must also be taken with the treatment of elderly patients and in making dosing decisions in the United States versus abroad.

Overall, capecitabine presents a promising step forward in transitioning treatment from infusional therapy to oral therapy, thereby limiting the time a patient with cancer must spend in clinic. More robust quality of life data would help to reinforce this claim. However, care must be taken to ensure that there is adequate information to truly support off-label use of expensive oncologic drugs; ongoing trials will be critical in supporting clinical decisions.

\section{Disclosure}

The authors report no conflicts of interest in this work.

\section{References}

1. Jemal A, Siegel R, Xu J, Ward E. Cancer statistics, 2010. CA Cancer J Clin. 2010;60(5):277-300.

2. Johnston PG, Kaye S. Capecitabine: a novel agent for the treatment of solid tumors. Anticancer Drugs. 2001;12(8):639-646.

3. National Comprehensive Cancer Network. The NCCN Clinical Practice Guidelines in Oncology: Colon Cancer (Version 2.2011). Available from: http://www.nccn.org/professionals/physician_gls/recently_updated.asp. Accessed November 29, 2010.

4. National Comprehensive Cancer Network. The NCCN Clinical Practice Guidelines in Oncology: Rectal Cancer (Version 2.2011). Available from: http://www.nccn.org/professionals/physician_gls/recently_updated.asp. Accessed November 29, 2010.

5. Tanaka M, Yoshida S, Saneyoshi M, Yamaguchi T. Utilization of 5-fluoro-2'-deoxyuridine triphosphate and 5-fluoro-2'-deoxycytidine triphosphate in DNA synthesis by DNA polymerases alpha and beta from calf thymus. Cancer Res. 1981;41(10):4132-4135.

6. Matsuoka H, Ueo H, Sugimachi K, Akiyoshi T. Preliminary evidence that incorporation of 5-fluorouracil into RNA correlates with antitumor response. Cancer Invest. 1992;10(4):265-269.

7. Miwa M, Ura M, Nishida M, et al. Design of a novel oral fluoropyrimidine carbamate, capecitabine, which generates 5-fluorouracil selectively in tumours by enzymes concentrated in human liver and cancer tissue. Eur J Cancer. 1998;34(8):1274-1281.

8. Schüller J, Cassidy J, Dumont E, et al. Preferential activation of capecitabine in tumor following oral administration to colorectal cancer patients. Cancer Chemother Pharmacol. 2000;45(4):291-297.

9. Sawada N, Ishikawa T, Fukase Y, Nishida M, Yoshikubo T, Ishitsuka H. Induction of thymidine phosphorylase activity and enhancement of capecitabine efficacy by taxol/taxotere in human cancer xenografts. Clin Cancer Res. 1998;4(4):1013-1019. 
10. Endo M, Shinbori N, Fukase Y, et al. Induction of thymidine phosphorylase expression and enhancement of efficacy of capecitabine or 5'-deoxy-5fluorouridine by cyclophosphamide in mammary tumor models. Int $J$ Cancer. 1999;83(1):127-134.

11. Morita T, Tokue A. Biomodulation of 5-fluorouracil by interferonalpha in human renal carcinoma cells: relationship to the expression of thymidine phosphorylase. Cancer Chemother Pharmacol. 1999;44(2): 91-96.

12. Sawada N, Ishikawa T, Sekiguchi F, Tanaka Y, Ishitsuka H. X-ray irradiation induces thymidine phosphorylase and enhances the efficacy of capecitabine (Xeloda) in human cancer xenografts. Clin Cancer Res. 1999;5(10):2948-2953.

13. Reigner B, Blesch K, Weidekamm E. Clinical pharmacokinetics of capecitabine. Clin Pharmacokinet. 2001;40(2):85-104.

14. Mackean M, Planting A, Twelves C, et al. Phase I and pharmacologic study of intermittent twice-daily oral therapy with capecitabine in patients with advanced and/or metastatic cancer. J Clin Oncol. 1998;16(9):2977-2985.

15. Reigner B, Watanabe T, Schüller J, et al. Pharmacokinetics of capecitabine (Xeloda) in Japanese and Caucasian patients with breast cancer. Cancer Chemother Pharmacol. 2003;52(3):193-201.

16. Judson IR, Beale PJ, Trigo JM, et al. A human capecitabine excretion balance and pharmacokinetic study after administration of a single oral dose of 14C-labelled drug. Invest New Drugs. 1999;17(1):49-56.

17. Reigner B, Verweij J, Dirix L, et al. Effect of food on the pharmacokinetics of capecitabine and its metabolites following oral administration in cancer patients. Clin Cancer Res. 1998;4(4):941-948.

18. Haller DG, Cassidy J, Clarke SJ, et al. Potential regional differences for the tolerability profiles of fluoropyrimidines. J Clin Oncol. 2008;26(13): 2118-2123.

19. Hochster HS, Hart LL, Ramanathan RK, et al. Safety and efficacy of oxaliplatin and fluoropyrimidine regimens with or without bevacizumab as first-line treatment of metastatic colorectal cancer: results of the TREE Study. J Clin Oncol. 2008;26(21):3523-3529.

20. Midgley R, Kerr DJ. Capecitabine: have we got the dose right? Nat Clin Pract Oncol. 2009;6(1):17-24.

21. Twelves C, Wong A, Nowacki MP, et al. Capecitabine as adjuvant treatment for stage III colon cancer. $N$ Engl J Med. 2005;352(26): 2696-2704.

22. Haller DG, Cassidy J, Tabernero J, et al. Efficacy findings from a randomized phase III trial of capecitabine plus oxaliplatin versus bolus 5-FU/LV for stage III colon cancer (NO16968): no impact of age on disease-free survival (DFS) [abstract 284]. Proceedings of the Gastrointestinal Cancers Symposium; 2010 Oct 1-3; Washington, DC, USA.

23. Roche Investor Update 2010. Roche provides results on Avastin in adjuvant colon cancer. Available from: http://www.roche.com/investors/ ir_update/inv-update-2010-09-18b.htm. Accessed November 29, 2010.

24. Glynne-Jones R, Dunst J, Sebag-Montefiore D. The integration of oral capecitabine into chemoradiation regimens for locally advanced rectal cancer: how successful have we been? Ann Oncol. 2006;17(3): 361-371.

25. National Cancer Institute. Clinical Trials. Phase III randomized study of adjuvant chemotherapy comprising capecitabine and oxaliplatin versus standard follow-up care in patients with clear margins after complete resection of locally advanced rectal cancer. Available from: http://www.cancer.gov/clinicaltrials/CRUK-CHRONICLE. Accessed November 29, 2010.

26. Sauer R, Becker H, Hohenberger W, et al. German Rectal Cancer Study Group. Preoperative versus postoperative chemoradiotherapy for rectal cancer. N Engl J Med. 2004;351(17):1731-1740.

27. De Paoli A, Chiara S, Luppi G, et al. Capecitabine in combination with preoperative radiation therapy in locally advanced, resectable, rectal cancer: a multicentric phase II study. Ann Oncol. 2006;17(2):246-251.

28. Krishnan S, Janjan NA, Skibber JM, et al. Phase II study of capecitabine (Xeloda) and concomitant boost radiotherapy in patients with locally advanced rectal cancer. Int J Radiat Oncol Biol Phys. 2006;66(3): $762-771$.
29. Kim JS, Kim JS, Cho MJ, Song KS, Yoon WH. Preoperative chemoradiation using oral capecitabine in locally advanced rectal cancer. Int J Radiat Oncol Biol Phys. 2002;54(2):403-408.

30. Kim JC, Kim TW, Kim JH, et al. Preoperative concurrent radiotherapy with capecitabine before total mesorectal excision in locally advanced rectal cancer. Int J Radiat Oncol Biol Phys. 2005;63(2): 346-353.

31. Hofheinz R, Wenz F, Post S, et al. Capecitabine (Cape) versus 5-fluorouracil (5-FU)-based (neo-)adjuvant chemoradiotherapy (CRT) for locally advanced rectal cancer (LARC): safety results of a randomized, phase III trial [abstract 4014]. J Clin Oncol. 2009;27(15S): 4014.

32. Rödel C, Liersch T, Hermann RM, et al. Multicenter phase II trial of chemoradiation with oxaliplatin for rectal cancer. J Clin Oncol. 2007;25(1):110-117.

33. Chua YJ, Barbachano Y, Cunningham D, et al. Neoadjuvant capecitabine and oxaliplatin before chemoradiotherapy and total mesorectal excision in MRI-defined poor-risk rectal cancer: a phase 2 trial. Lancet Oncol. 2010;11(3):241-248.

34. Gérard JP, Azria D, Gourgou-Bourgade S, et al. Comparison of two neoadjuvant chemoradiotherapy regimens for locally advanced rectal cancer: results of the phase III trial ACCORD 12/0405-Prodige 2. J Clin Oncol. 2010;28(10):1638-1644.

35. Machiels JP, Duck L, Honhon B, et al. Phase II study of preoperative oxaliplatin, capecitabine and external beam radiotherapy in patients with rectal cancer: the RadiOxCape study. Ann Oncol. 2005;16(12): 1898-1905.

36. Rödel C, Grabenbauer GG, Papadopoulos T, Hohenberger W, Schmoll HJ, Sauer R. Phase I/II trial of capecitabine, oxaliplatin, and radiation for rectal cancer. J Clin Oncol. 2003;21(16):3098-3104.

37. Willeke F, Horisberger K, Kraus-Tiefenbacher U, et al. A phase II study of capecitabine and irinotecan in combination with concurrent pelvic radiotherapy (CapIri-RT) as neoadjuvant treatment of locally advanced rectal cancer. Br J Cancer. 2007;96(6):912-917.

38. Klautke G, Küchenmeister U, Foitzik T, et al. Concurrent chemoradiation with capecitabine and weekly irinotecan as preoperative treatment for rectal cancer: results from a phase I/II study. Br J Cancer. 2006; 94(7):976-981.

39. Machiels JP, Sempoux C, Scalliet P, et al. Phase I/II study of preoperative cetuximab, capecitabine, and external beam radiotherapy in patients with rectal cancer. Ann Oncol. 2007;18(4):738-744.

40. Rödel C, Arnold D, Hipp M, et al. Phase I-II trial of cetuximab, capecitabine, oxaliplatin, and radiotherapy as preoperative treatment in rectal cancer. Int J Radiat Oncol Biol Phys. 2008;70(4):1081-1086.

41. Twelves C, Gollins S, Grieve R, Samuel L. A randomised crossover trial comparing patient preference for oral capecitabine and 5 -fluorouracil/leucovorin regimens in patients with advanced colorectal cancer. Ann Oncol. 2006;17(2):239-245.

42. Liu G, Franssen E, Fitch MI, Warner E. Patient preferences for oral versus intravenous palliative chemotherapy. J Clin Oncol. 1997;15(1): $110-115$.

43. Ling W, Fan J, MaY, Ma Y, Wang H. Capecitabine-based chemotherapy for metastatic colorectal cancer. J Cancer Res Clin Oncol. 2010. DOI: 10.1007/s00432-010-0954-0.

44. Hoff PM, Ansari R, Batist G, et al. Comparison of oral capecitabine versus intravenous fluorouracil plus leucovorin as first-line treatment in 605 patients with metastatic colorectal cancer: results of a randomized phase III study. J Clin Oncol. 2001;19(8):2282-2292.

45. Van Cutsem E, Twelves C, Cassidy J, et al. Xeloda Colorectal Cancer Study Group. Oral capecitabine compared with intravenous fluorouracil plus leucovorin in patients with metastatic colorectal cancer: results of a large phase III study. J Clin Oncol. 2001;19(21): 4097-4106.

46. Cassidy J, Clarke S, Díaz-Rubio E, et al. Randomized phase III study of capecitabine plus oxaliplatin compared with fluorouracil/folinic acid plus oxaliplatin as first-line therapy for metastatic colorectal cancer. J Clin Oncol. 2008;26(12):2006-2012. 
47. Porschen R, Arkenau HT, Kubicka S, et al; AIO Colorectal Study Group. Phase III study of capecitabine plus oxaliplatin compared with fluorouracil and leucovorin plus oxaliplatin in metastatic colorectal cancer: a final report of the AIO Colorectal Study Group. J Clin Oncol. 2007;25(27):4217-4223.

48. Díaz-Rubio E, Tabernero J, Gómez-España A, et al; Spanish Cooperative Group for the Treatment of Digestive Tumors Trial. Phase III study of capecitabine plus oxaliplatin compared with continuous-infusion fluorouracil plus oxaliplatin as first-line therapy in metastatic colorectal cancer: final report of the Spanish Cooperative Group for the Treatment of Digestive Tumors Trial. J Clin Oncol. 2007;25(27):4224-4230.

49. Ducreux M, Bennouna J, Hebbar M, et al. Efficacy and safety findings from a randomized phase III study of capecitabine $(\mathrm{X})+$ oxaliplatin (O) (XELOX) vs infusional 5-FU/LV + O (FOLFOX-6) for metastatic colorectal cancer (MCRC) [abstract 4029]. J Clin Oncol. 2007;25(18S):4029.

50. Rothenberg ML, Cox JV, Butts C, et al. Capecitabine plus oxaliplatin (XELOX) versus 5-fluorouracil/folinic acid plus oxaliplatin (FOLFOX-4) as second-line therapy in metastatic colorectal cancer: a randomized phase III noninferiority study. Ann Oncol. 2008;19(10): $1720-1726$.

51. Koopman M, Antonini NF, Douma J, et al. Sequential versus combination chemotherapy with capecitabine, irinotecan, and oxaliplatin in advanced colorectal cancer (CAIRO): a phase III randomised controlled trial. Lancet. 2007;370(9582):135-142.

52. Fuchs CS, Marshall J, Mitchell E, et al. Randomized, controlled trial of irinotecan plus infusional, bolus, or oral fluoropyrimidines in firstline treatment of metastatic colorectal cancer: results from the BICC-C study. J Clin Oncol. 2007;25(30):4779-4786.

53. Köhne CH, De Greve J, Hartmann JT, et al. Irinotecan combined with infusional 5-fluorouracil/folinic acid or capecitabine plus celecoxib or placebo in the first-line treatment of patients with metastatic colorectal cancer. EORTC study 40015. Ann Oncol. 2008;19(5):920-926.

54. Walko CM, Lindley C. Capecitabine: a review. Clin Ther. 2005;27(1): 23-44.
55. Díaz-Rubio E, Burris H, Douillard JY, et al; The X-Act Study Group. Safety of capecitabine $(\mathrm{X})$ compared to fluorouracil/leucovorin (5-FU/LV) for the adjuvant treatment of elderly colon cancer patients (pts) [abstract 3737]. J Clin Oncol. 2004;22(14S):3737.

56. Feliu J, Escudero P, Llosa F, et al. Capecitabine as first-line treatment for patients older than 70 years with metastatic colorectal cancer: an oncopaz cooperative group study. J Clin Oncol. 2005;23(13):3104-3111.

57. Feliu J, Safont MJ, Salud A, et al. Capecitabine and bevacizumab as first-line treatment in elderly patients with metastatic colorectal cancer. Br J Cancer. 2010;102(10):1468-1473.

58. Rosati G, Cordio S, Bordonaro R, et al. Capecitabine in combination with oxaliplatin or irinotecan in elderly patients with advanced colorectal cancer: results of a randomized phase II study. Ann Oncol. 2010;21(4):781-786.

59. Hennig IM, Naik JD, Brown S, et al. Severe sequence-specific toxicity when capecitabine is given after fluorouracil and leucovorin. J Clin Oncol. 2008;26(20):3411-3417.

60. Chu E, Schulman KL, Zelt S, Song X. Costs associated with complications are lower with capecitabine than with 5-fluorouracil in patients with colorectal cancer. Cancer. 2009;115(7):1412-1423.

61. Cassidy J, Douillard JY, Twelves C, et al. Pharmacoeconomic analysis of adjuvant oral capecitabine vs intravenous 5-FU/LV in Dukes' $\mathrm{C}$ colon cancer: the X-ACT trial. Br J Cancer. 2006;94(8):1122-1129.

62. Shiroiwa T, Fukuda T, Tsutani K. Cost-effectiveness analysis of XELOX for metastatic colorectal cancer based on the NO16966 and NO16967 trials. Br J Cancer. 2009;101(1):12-18.

63. Chu E, Schulman KL, McKenna EF Jr, Cartwright T. Patients with locally advanced and metastatic colorectal cancer treated with capecitabine versus 5-fluorouracil as monotherapy or combination therapy with oxaliplatin: a cost comparison. Clin Colorectal Cancer. 2010;9(4): 229-237.

64. Velenik V, Ocvirk J, Omejc M, et al. Neoadjuvant bevacizumab, capecitabine, and radiotherapy in locally advanced rectal cancer: interim results of a phase II CRAB trial [abstract e14006]. J Clin Oncol. 2010;28(15) e14006.
Cancer Management and Research

\section{Publish your work in this journal}

Cancer Management and Research is an international, peer-reviewed open access journal focusing on cancer research and the optimal use of preventative and integrated treatment interventions to achieve improved outcomes, enhanced survival and quality of life for the cancer patient The journal welcomes original research, clinical \& epidemiological

\section{Dovepress}

studies, reviews \& evaluations, guidelines, expert opinion \& commentary, case reports \& extended reports. The manuscript management system is completely online and includes a very quick and fair peerreview system, which is all easy to use. Visit http://www.dovepress.com/ testimonials.php to read real quotes from published authors. 